\title{
Physiological Characteristics of Indonesian Junior Badminton Players: Men's Double Category
}

\author{
Tommy Apriantono ${ }^{1, *}$, Indria Herman ${ }^{2}$, Bagus Winata ${ }^{1}$, Iwa Ikhwan Hidayat ${ }^{1}$, \\ Muhamad Fahmi Hasan ${ }^{1}$, Agung Dwi Juniarsyah ${ }^{1}$, Sri Indah Ihsani ${ }^{1}$
}

\begin{abstract}
${ }^{1}$ Department of Sport Science, Faculty of Pharmacy, Bandung Institute of Technology, Kota Bandung, Jawa Barat 40132, Indonesia ${ }^{2}$ Faculty of Mechanical and Aerospace Engineering, Bandung Institute of Technology, Kota Bandung, Jawa Barat 40132, Indonesia
\end{abstract}

Received October 11, 2020; Revised November 27, 2020; Accepted December 13, 2020

\section{Cite This Paper in the following Citation Styles}

(a): [1] Tommy Apriantono, Indria Herman, Bagus Winata, Iwa Ikhwan Hidayat, Muhamad Fahmi Hasan, Agung Dwi Juniarsyah, Sri Indah Ihsani , "Physiological Characteristics of Indonesian Junior Badminton Players: Men's Double Category," International Journal of Human Movement and Sports Sciences, Vol. 8, No. 6, pp. 444 - 454, 2020. DOI: 10.13189/saj.2020.080617.

(b): Tommy Apriantono, Indria Herman, Bagus Winata, Iwa Ikhwan Hidayat, Muhamad Fahmi Hasan, Agung Dwi Juniarsyah, Sri Indah Ihsani (2020). Physiological Characteristics of Indonesian Junior Badminton Players: Men's Double Category. International Journal of Human Movement and Sports Sciences, 8(6), 444 - 454. DOI: 10.13189/saj.2020.080617.

Copyright $\subseteq 2020$ by authors, all rights reserved. Authors agree that this article remains permanently open access under the terms of the Creative Commons Attribution License 4.0 International License

Abstract The primary aim of this study is to assess the $\mathrm{VO}_{2 \max }$ and anthropometric characteristics of men's double category; Next is to analyze match characteristics of men's double category; and finally, to compare the heart rate (HR) and blood lactate concentration calculated on-court with that obtained under laboratory conditions during an incremental test. 12 junior badminton players from Jaya Raya Badminton Club were enrolled as the subjects for this study. All participants were partnered up in 6 pairs (pair 1 to pair 6). During the first session (laboratory condition), $\mathrm{VO}_{2 \max }$ was measured by using a velocity dependent ramp test (INCS) based on incremental protocols. During the second session (on-court condition), the participants played a simulated match on court. A total of 350 rallies were analyzed. Statistical analyses revealed that double junior men's players hit the shuttlecock more often using drive shots techniques (466 shots). The ANOVA revealed significant differences regarding $f_{B}$ between pair 3 vs pair 6 $(\mathrm{p}=0.004)$, pair 4 vs pair $6(\mathrm{p}=0.030)$, and pair 5 vs pair 6 $(\mathrm{p}=0.023)$. For the lactate measures, ANOVA revealed significant main effects of time $(\mathrm{p}=0.001)$ and group $(p=0.001)$. Furthermore, the significance of HR average among laboratory and on-court conditions was established with the help of an independent t-test. The study demonstrated that the physiological characteristics of Indonesian men's double category are intermittent activities of high and low intensities, interspersed by short recovery periods. It was also discovered that the adrenergic strategy results in enhanced HR and lactate levels in the laboratory than on-court conditions.

Keywords Physiological Profile, Racket Sport, Laboratory Measurements, On-Court Measurements, Performance

\section{Introduction}

Since its introduction as an Olympic game in 1992, badminton has gained huge popularity all over the world [1]. The growing popularity of the sport worldwide has made researchers eager to study and examine the physiological characteristics of badminton match play [2]. For example, Jeyaraman et al. [3] observed that performance in badminton is characterized by intermittent activities of high and low intensities, interspersed by short recovery periods. Another study explained that during the performance of the explosive movements (e.g. lunges, smash) in each rally, anaerobic actions play an important role. The aerobic system provides about $65 \%$ of the energy needed for the continuous alternation of high 
intensity rallies, short recovery periods, and moderate intensity activities [4]. Despite the fact that some studies have succeeded in disseminating the physiological characteristics of badminton, research on the sport is still scarce, even more so when compared to the others sports.

Badminton has five categories: men's singles, women's singles, men's doubles, women's doubles, and mixed doubles. Each category has different physical demands and match play characteristics [2]. This is clarified by several previous studies, that reported differences in physiological demands and match characteristics between two disciplines (single and doubles category) [5,6]. For instance, Alcock et al. [5] demonstrated that $\mathrm{VO}_{2 \max }$ of singles players had greater value than double players (50.6 vs. $45.5 \mathrm{~mL} / \mathrm{kg} / \mathrm{min}$ ). On the other hand, Liddle et al. [6] analysed that match characteristics in single-player rallies are more demanding than doubles with approximately $80 \%$ of the rallies lasting less than 10 seconds. In addition, previous studies have also established the existence of differences in physical demands based on the gender of the players (male or female) [7].

The men's doubles category, one of the five events played in badminton [8], is composed of two opposing pairs of male players [5]. Similar to other categories, men's doubles is a match that consists of the best of three games of twenty-one points [9]. Research specifically examining physiological characteristics of badminton during men's doubles is insufficient in nature, when compared to studies that examine general badminton physiological characteristics $[10,11]$. It was also found that most of the previous studies on badminton only focused on senior players [12,13,14], resulting in limited information regarding badminton junior players. This had led to indifferent evaluation of training methods in case of badminton junior athletes.

Previous studies have generally focused on examining the physiological demands and match characteristics in different settings such as laboratory and on the field, obtaining the $\mathrm{VO}_{2 \max }$ and match characteristics (average shots and rally time) of badminton athletes [15]. Consequently, many sports scientists and coaches have utilized the research findings to determine the evidence-based design of training regimens [16-19]. For example, previous research conducted by Cabello et al. [16] concluded that badminton is characterized by repetitive efforts of lactic nature and great intensity, which are continuously performed throughout the match. The results suggested that coaches should devise a training regimen concentrating on a large number of competitive actions of high intensity but short duration. Additionally, Cabello et al. [16] recommended that badminton athletes should train to develop specific endurance skills by means of actions and moves performed at short (15-20 seconds) and very short (6-10 seconds) intervals.

Susanna Rampichini et al. [17] explained that the difference in heart rate (HR) $\mathrm{vs} \mathrm{VO}_{2}$ regression lines between the laboratory examination (IIAT test) and the on-court condition indicated that HR monitoring may not be sufficient in providing accurate data on the aerobic demands of specific on-court badminton tasks. The findings of these studies are equivocal in nature concerning the general physiological characteristics of badminton athletes in laboratory and on-court badminton tasks. Hence, the results that are used to formulate appropriate planning and monitoring training structures are lacking.

To the authors' best knowledge, no available studies have reported the specific measurements of junior men's doubles category. On the basis of these problems and interests, the primary aim of this study is to assess the $\mathrm{VO}_{2 \max }$ and anthropometric characteristics of men's double category; Next is to analyze match characteristics of men's double category; and finally, to compare the heart rate (HR) and blood lactate concentration calculated on-court with that obtained under laboratory conditions during an incremental test. Furthermore, the findings of this study can add to the scientific literature that supports the use of physiological characteristics as a formula to determine training regimens concerning badminton, that can be used by coaches, sports scientists, and junior badminton athletes, specifically for men's doubles category.

\section{Materials and Methods}

In this observational study, 12 male junior badminton players from Jaya Raya Badminton Club were recruited based on the following criteria: minimum continuous badminton training background of 5 years as a men's doubles category athlete, national or international level tournament participation, 15 or more hours of training per week, current and past non-smokers, no concomitant diseases, and no use of any antioxidant or anti-inflammatory drugs during and one month prior to the experimental period. None of the participants had lower and upper-extremity injuries or musculoskeletal injuries within the last 6 months, prior to the initial testing. All participants provided informed consent to participate in the observational study and experimental procedures. All of the procedures were approved by the ethics committee of the Bandung Health Polytechnic (02/KEPK/PE/XI/2018). 


\subsection{Study Design}

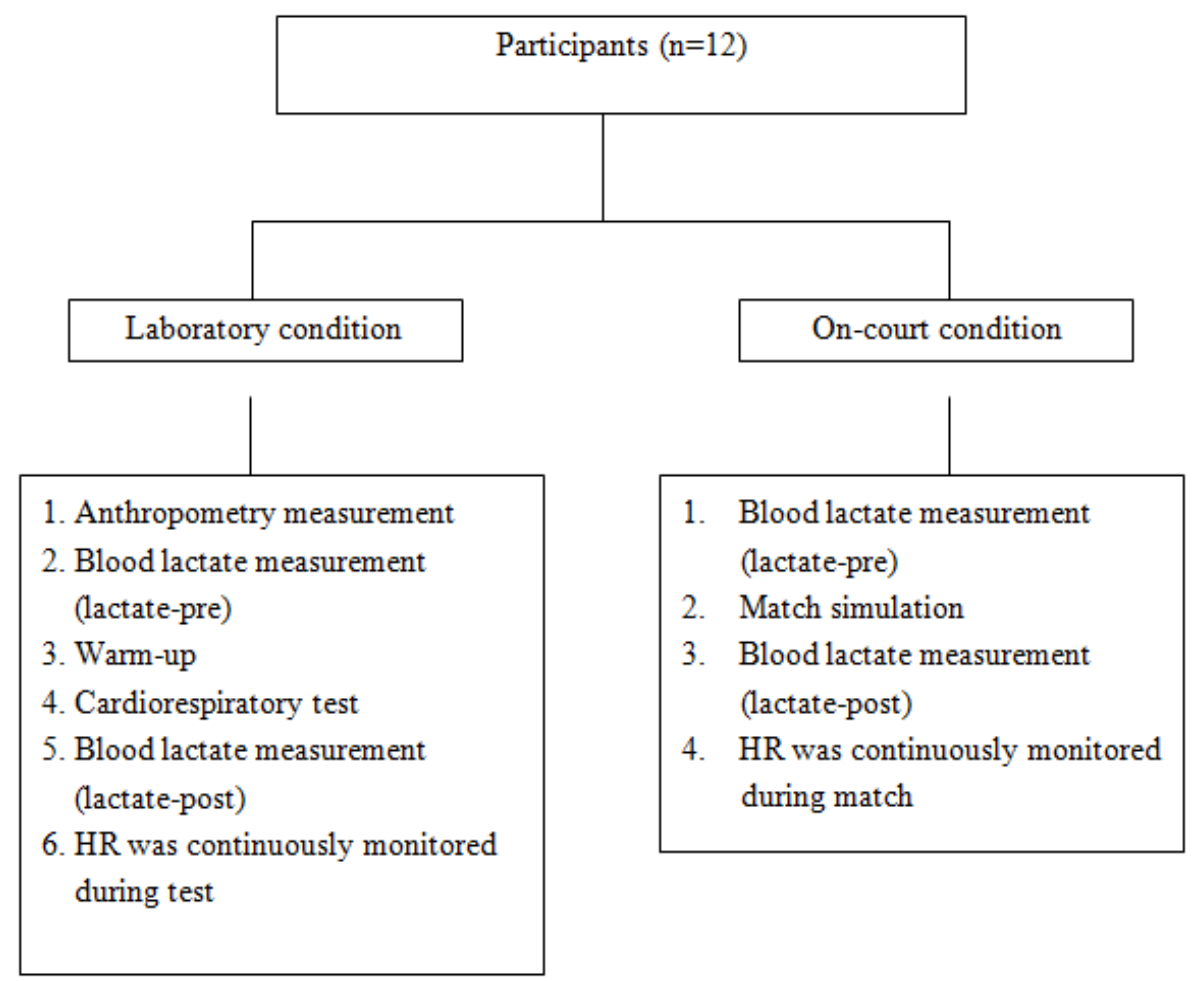

Figure 1. The research designs

This study requires all participants to complete one familiarization session and two experimental sessions. All participants are partnered up into 6 pairs (pair 1, pair 2, pair 3, pair 4, pair 5, pair 6) and they successfully completed two experimental sessions. During the first session (laboratory condition), $\mathrm{VO}_{2 \max }$ was measured by using a velocity dependent ramp test (INCS) based on incremental protocols. During the second session (on-court condition), the participants played a simulated match on court in accordance with the current set of rules published by Badminton World Federation (BWF). Both the tests were separated by a week of recovery period. The research design is illustrated in Figure 1.

\subsection{Laboratory Condition}

Before undergoing the test, participants were asked to have a light dinner (before 09:00 p.m.) the day before, and not to eat anything or drink caffeine beverages on the test day. The initial anthropometric characteristics were measured in laboratory on Monday morning at 08:00 a.m. The anthropometry and cardiorespiratory tests were conducted at constant ambient temperature $\left(27-29^{\circ} \mathrm{C}\right)$ and relative humidity $(64-74 \%)$. The body weight of the participants (wearing minimal clothes and barefoot) was measured on Omron Digital Weight Scale HN 289. The height of the participants was measured with the help of a stadiometer with $0.1 \mathrm{~cm}$ readability (Seca 214 Portable Stadiometer, Cardinal Health, Ohio, USA). Further, the body scan composition was measured using Dexa (DXA) Scan Body Fat, according to the described standardised procedures.

Following the anthropometric measurements, $100 \mu \mathrm{L}$ sample of capillary blood was obtained from the fingertips of the participants to measure lactate. The blood samples were analysed with the help of a Lactate Pro analyser (Arkray, Shiga, Japan). Prior to cardiorespiratory-testing, all participants were required to complete Electro Cardio Graph (ECG) process, in order to monitor their cardiac normalities. After the ECG session, participants performed a warm-up practice (6 min jog at $6.8 \mathrm{~km} \mathrm{~h}^{-1}$ ). The cardiorespiratory test began at 09:30 a.m. and was conducted using a treadmill cardio pulmonary exercise inspection. The Quark CPET T170 Compact metabolic cart with breath by breath gas exchange analysis helped us obtain the values of each player's maximum heart rate, minute ventilation $\left(\mathrm{V}_{\mathrm{E}}\right)$, respiratory frequency $\left(\mathrm{f}_{\mathrm{B}}\right)$ and $\mathrm{VO}_{2 \max }$. The $\mathrm{VO}_{2 \max }$ was measured by using a velocity dependent ramp test $\left(\mathrm{INC}_{\mathrm{S}}\right)$ on the basis of incremental protocols, at constant inclination $\left(0^{\circ}\right)$, and increasing speed $\left(0.5 \mathrm{~km} \cdot \mathrm{h}^{-1}\right.$ every $30 \mathrm{~s}$ from the initial $\left.12.0 \mathrm{~km} \cdot \mathrm{h}^{-1}\right)$. $\mathrm{VO}_{2 \max }$ is defined as the plateau attained by the relationship between $\mathrm{VO}_{2}$ and running speed. Participants were verbally encouraged until volitional exhaustion. The heart rate (HR) was continuously monitored employed a Polar V800 GPS. At the end of each workload, the rate of perceived exertion (RPE) was evaluated by using the CR-10 scale as described by Foster (1998) [20]. At the 
end of the last task, blood lactate was collected during the $3^{\text {rd }}$ minute after undergoing the cardiorespiratory test. Participants were allowed to drink ad libitum mineral water during the recovery period, but we encouraged them to drink only enough to maintain hydration.

\subsection{On- court Condition}

The on-court condition testing was conducted in the Badminton Jaya Raya Stadium Jakarta a week after finishing the laboratory condition tests. All participants lived in the athletes' dormitories and followed the same training sessions prior to on-court condition-testing. Three badminton matches were played in accordance with the current set of rules devised by the Badminton World Federation (BWF) (https://corporate.bwfbadminton.com/ statutes/\#1513733305001-7485aaef-d176). To ensure that the athletes were highly motivated, the matches were included in the regular internal ranking competition. A video camera (Handycam Canon LEGRIA FS200) was mounted 5-m behind the baseline and 4-m above the ground at the same end of each court to film each simulated match. A $100 \mu \mathrm{L}$ sample of capillary blood was also obtained from the fingertips of the athletes pre-match and post-match (immediately after simulation match) in order to measure lactate. Furthermore, the HR was also continuously monitored during the simulation matches with Polar RS400 Finland. To ensure that the participants were giving their maximum effort during the matches, the players were asked to provide a RPE using Foster's 0-10 scale at the end of each match.

The technical assessment during match simulations was developed by members of the training team who have more than 10 years of experience in badminton. The assessment of match characteristics was adopted based on previous assessment method [16]; (i) The smash is an aggressive overhead shot with a downward trajectory, (ii) the clear is an overhead shot with a flat (offensive clear) or rising trajectory (defensive clear) towards the back of the opponent's court, (iii) the drop is a smooth shot performed above the head with a downward trajectory towards the front of the court, (iv) the net shot is a precise shot taken near the net, which includes the net drop, (v) the lob (offensive shot with a flat trajectory towards the back of the opponent's court and defensive shot with a rising trajectory) and the kill (aggressive shot with downward trajectory), (vi) the drive is a powerful shot performed at mid-body height and in the middle of the court with a flat trajectory, (vii) a forced error occurs if a player is unable to make a reasonable attempt at playing a shot and the shuttlecock does not land in the opposition's court, and (viii) an unforced error occurs when a participant has adequate time and space to play a shot but misses the court (either outside the lines or into the net). The match design is displayed in Figure 2.

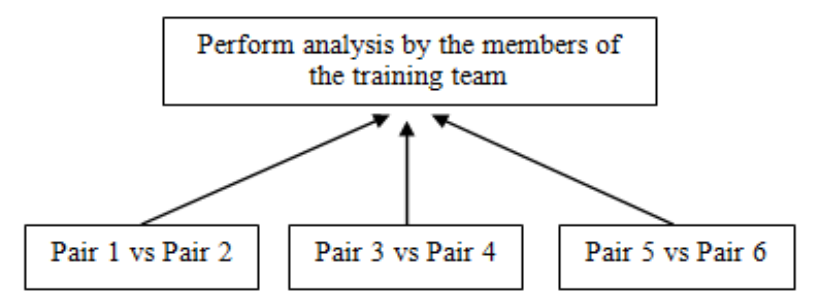

Figure 2. Head to head badminton match simulation

\section{4. $\mathrm{VO}_{2 \max }$ Measurements}

The validity of the instrument was confirmed by comparing the Quark CPET T170 testing protocol with Douglas-bag and iron lung (Cortex, Leipzig, Germany) 2 days before the commencement of the INCS examination. All devices contained in the Quark CPET T170 utilized in this study are new apparatus that were tested and found both reliable and valid. Before starting the measurement process for each participant, the Quark CPET T170 was calibrated using a predetermined factory validation standard, employing a metabolic calibrator capable of sending a cyclic air flow of tidal volume, frequency, and known gas composition [21,22]. All subjects used a head gear attached to a rubber mouthpiece, a turbine flow meter, a two-way nonrebreathing valve (Hans-Rudolph Inc., Kansas City, MO) plastic hose for the transfer of expired air to a 7-litre mixing chamber, and a sampling line connected from the mixing chamber to CPET gas analysers. The specified range for a paramagnetic oxygen analyser is $0 \%-25 \%$, for the analysis of fraction of air as oxygen, while $0 \%$ to $10 \%$ is the range value set on a non-dispersive infrared sensor (NDIR) carbon dioxide analyser to analyse carbon dioxide. Measurement of flow rate is done using a two-way digital turbine that has an accuracy value of $\pm 2 \%$, with a range of 0.08 to $20 \mathrm{~L} /$ second, and a range of 0 to $300 \mathrm{~L} / \mathrm{min}$, which is used as the range value for $\mathrm{VE}$ measurements during the testing process. The 7-litre mixing chamber allowed metabolic assessment through a full range of low to high VE rates during exercise testing.

For the DXA body scan measurement, all participants were instructed to wear as few clothes as possible. DXA is carried out on the principle of using two X-ray attenuators that pass through each participant's body to accurately calculate the masses of two different objects based on simple algebraic principles and physical properties of the objects. DXA can measure body thickness with an accuracy of $25 \mathrm{~cm}$ to $30 \mathrm{~cm}$, and with a precision level better than 1\%. For instance, one skeleton was measured by using 37 systems and the coefficient of variation (intrascanner) was found to be $0.96 \%$. The DXA splits into several areas, such as arms, legs, trunk, and head. Fat, lean soft tissue, and bone mass composition were reported for each subregion. 


\section{Statistical Analysis}

Normal distribution of the sample was checked by using the Shapiro-Wilk test. One-way repeated measures ANOVA with Tukey post-hoc test was performed to obtain the anthropometric (weight, height, BMI, body fat), cardiorespiratory $\left(\mathrm{VO}_{2 \max }, \mathrm{VE}, \mathrm{Rf}\right)$ and rally characteristic parameters among the pair of participants. A 2 (condition tests: laboratory and match simulation) $\times 2$ (time: pre, post) repeated measures analysis of variance (ANOVA) was calculated for the lactate concentration of all participants. An independent t-test was used to determine any differences in HR among the laboratory and match simulation tests. The values are presented as mean \pm SD. Statistical significance was accepted at the $p<0.05$ level.

\section{Results}

\subsection{Laboratory Condition}

The averages of anthropometric and cardiorespiratory characteristics of the study sample are presented in Table 1; and Table 2 presents the differences in anthropometric (weight, height, BMI, body fat) and cardiorespiratory characteristics $\left(\mathrm{VO}_{2 \max }, \mathrm{V}_{\mathrm{E}}, \mathrm{f}_{\mathrm{B}}\right)$ among each pair of participants. Regarding $\mathrm{V}_{\mathrm{E}}$, statistically significant differences were found among pair 1 vs pair $3(p=0.045)$. For the $f_{B}$, ANOVA revealed significant differences between pair 3 vs pair $6(p=0.004)$, pair 4 vs pair 6 $(p=0.030)$, as well as pair 5 vs pair $6(p=0.023)$.

Table 1. Anthropometric data and performance characteristics of Participants

\begin{tabular}{cc}
\hline Variables & $\overline{\boldsymbol{X}}(\boldsymbol{S D})$ \\
\hline Age (years) & $16.50( \pm 0.67)$ \\
\hline Height $(\mathrm{cm})$ & $175.3( \pm 5.93)$ \\
\hline Weight $(\mathrm{kg})$ & $69.01( \pm 7.55)$ \\
\hline BMI $(\mathrm{kg} / \mathrm{m})$ & $22.43( \pm 1.98)$ \\
\hline Body fat $(\mathrm{kg})$ & $13.02( \pm 3.80)$ \\
\hline Muscle mass in Humerus Dextra $(\mathrm{kg})$ & $3.36( \pm 0.43)$ \\
\hline Muscle mass in Humerus Sinistra $(\mathrm{kg})$ & $2.89( \pm 0.52)$ \\
\hline Muscle mass in Trunk Dextra $(\mathrm{kg})$ & $11.98( \pm 1.16)$ \\
\hline Muscle mass in Trunk Sinistra $(\mathrm{kg})$ & $12.43( \pm 1.05)$ \\
\hline Peak minute ventilation $\left(\mathrm{l} / \mathrm{min}^{-1}\right)$ & $119.21( \pm 18.84)$ \\
\hline Respiratory frequency $\left(\mathrm{min}^{-1}\right)$ & $57.76( \pm 12.03)$ \\
\hline Peak VO $/$ HR (ml/bpm) & $17.42( \pm 2.48)$ \\
\hline VO $_{2 \text { max }}(\mathrm{ml} / \mathrm{kg} / \mathrm{min})$ & $49.95( \pm 4.45)$ \\
\hline RPE CR-10 scale (laboratory condition) & $9.0( \pm 0.43)$ \\
\hline RPE CR-10 scale (on-court condition) & $8.08( \pm 0.79)$
\end{tabular}

Table 2. The differences in weight, height, BMI, body fat, VO2max, VE, fB measures on each pair of participants

\begin{tabular}{cccccccc}
\hline Pair & $\begin{array}{c}\text { Weight } \\
(\mathrm{kg})\end{array}$ & Height $(\mathrm{cm})$ & BMI $(\mathrm{kg} / \mathrm{m} 2)$ & Body fat $(\mathrm{kg})$ & $\begin{array}{c}\mathrm{VO}_{2 \max } \\
(\mathrm{ml} / \mathrm{kg} / \mathrm{min}-1)\end{array}$ & $\mathrm{V}_{\mathrm{E}}\left(\mathrm{l} / \mathrm{min}^{-1}\right)$ & $\mathrm{f}_{\mathrm{B}}\left(\mathrm{min}^{-1}\right)$ \\
\hline Pair 1 & $74.5 \pm 8.29$ & $174 \pm 12.73$ & $24.6 \pm 0.8$ & $15.02 \pm 3.1$ & $54.18 \pm 0.88$ & $136.65 \pm 7.57^{*}$ & $59.65 \pm 2.19$ \\
Pair 2 & $69.94 \pm 8.82$ & $180 \pm 1.41$ & $21.61 \pm 3.09$ & $14.17 \pm 7.27$ & $48.04 \pm 4.76$ & $126.62 \pm 23.44$ & $60.05 \pm 9.97$ \\
Pair 3 & $67.5 \pm 13.72$ & $174.5 \pm 7.78$ & $22.01 \pm 2.56$ & $12.42 \pm 5.05$ & $50.55 \pm 10.96$ & $95.25 \pm 23.55$ & $42.1 \pm 11.74^{\#}$ \\
Pair 4 & $67.44 \pm 10.97$ & $177 \pm 0$ & $21.54 \pm 3.49$ & $12.27 \pm 2.79$ & $47.56 \pm 0.35$ & $111.55 \pm 20.15$ & $55 \pm 9.48 \wedge$ \\
Pair 5 & $71.7 \pm 3.11$ & $178 \pm 0$ & $22.62 \pm 0.99$ & $13.92 \pm 5.29$ & $50.04 \pm 2.74$ & $114.55 \pm 1.34$ & $53.4 \pm 0.99^{\ddagger}$ \\
Pair 6 & $62.98 \pm 2.14$ & $168.5 \pm 0.71$ & $22.16 \pm 0.53$ & $10.33 \pm 2.52$ & $49.34 \pm 3.21$ & $130.65 \pm 6.72$ & $76.35 \pm 2.76$ \\
\hline
\end{tabular}

Abbreviations:

The values are presented as mean \pm SD.

The mean difference is significant at the $\mathrm{p}<0.05$ level.

*Significant difference among pair 1 and pair 3

\#Significant difference among pair 3 and pair 6

$\wedge$ Significant difference among pair 4 and pair 6

¥Significant difference among pair 5 and pair 6 


\subsection{On-court Condition}

A total 350 rallies in three matches were played. Table 3 displays the average characteristics of different shots taken in the three simulated badminton matches. Statistical analyses revealed that doubles junior men's players hit the shuttlecock most often with drive shots techniques (466 shots), subsequently with drop (337 shots) and lob shots (298 shots). The averages in case of smashes and net shots were found to be 168 and 25 shots, respectively. Furthermore, the match statistical analyses revealed that the number of unforced errors was higher than forced errors (114 vs 45 shots). For the rally characteristics of each pair (as presented in Table 4),
ANOVA discovered significant differences between pair 3 vs pair $6(p=0.016)$, pair 4 vs pair $6(p=0.029)$ in drive shots, and pair 1 vs pair $6(p=0.046)$ in smash shots. Additionally, the statistical analysis established significant values among pair 2 vs $4(p=0.038)$ and pair 2 vs 5 $(p=0.023)$ regarding forced error during match simulation.

The mean characteristics of badminton match play for all matches are presented in Figure 3. The most frequently occurring rallies in three matches were analysed to be $89 \%$ of all rallies, lasting for 11 seconds or less with a rally time between 3-8 seconds. Furthermore, just $11 \%$ of all rallies lasted longer than 12 seconds. The rest intervals were mostly set up between $4-11$ seconds $(81.6 \%$ of the three matches).

Table 3. The distribution of the different shots of three matches badminton simulation

\begin{tabular}{ccccccc}
\hline Lob & Drive & Smash & Drop & Net & Forced & Unforced \\
\hline 298 & 466 & 168 & 337 & 25 & 45 & 114 \\
\hline
\end{tabular}

Table 4. The rally characteristics of the study sample during match simulations

\begin{tabular}{cccccccc}
\hline Pair & Lob & Drive & Smash & Drop & Net & Forced Error & $\begin{array}{c}\text { Unforced } \\
\text { Error }\end{array}$ \\
\hline Pair 1 & $27 \pm 11.31$ & $38 \pm 8.49$ & $19.5 \pm 7.78^{*}$ & $25.5 \pm 4.95$ & $1.5 \pm 0.71$ & $3.5 \pm 0.71$ & $6.5 \pm 3.54$ \\
Pair 2 & $26 \pm 9.90$ & $40.5 \pm 0.71$ & $12.5 \pm 2.12$ & $24 \pm 5.66$ & $3.5 \pm 3.54$ & $6.5 \pm 0.71 \dagger^{\ddagger}$ & $12.5 \pm 2.12$ \\
Pair 3 & $30.5 \pm 2.12$ & $47 \pm 1.41^{\#}$ & $14.5 \pm 0.71$ & $32.5 \pm 3.54$ & $2.5 \pm 2.12$ & $3.5 \pm 0.71$ & $8 \pm 7.07$ \\
Pair 4 & $32.5 \pm 2.12$ & $44.5 \pm 0.71^{\wedge}$ & $15.5 \pm 2.12$ & $33.5 \pm 2.12$ & $2 \pm 2.83$ & $3 \pm 2.83$ & $10.5 \pm 7.78$ \\
Pair 5 & $18 \pm 9.90$ & $34.5 \pm 10.61$ & $12.5 \pm 3.54$ & $33 \pm 9.90$ & $1 \pm 1.41$ & $2.5 \pm 0.71$ & $10 \pm 2.83$ \\
Pair 6 & $15 \pm 2.83$ & $28.5 \pm 0.71$ & $9.5 \pm 3.54$ & $20 \pm 5.66$ & $2 \pm 2.83$ & $3.5 \pm 0.71$ & $9.5 \pm 6.36$ \\
\hline
\end{tabular}

Abbreviations:

The values are presented as mean \pm SD.

The mean difference is significant at the $\mathrm{p}<0.05$ level.

*Significant difference among pair 1 and pair 6

\#Significant difference among pair 3 and pair 6

$\wedge$ Significant difference among pair 4 and pair 6

†Significant difference among pair 2 and pair 4

$\neq$ Significant difference among pair 2 and pair 5

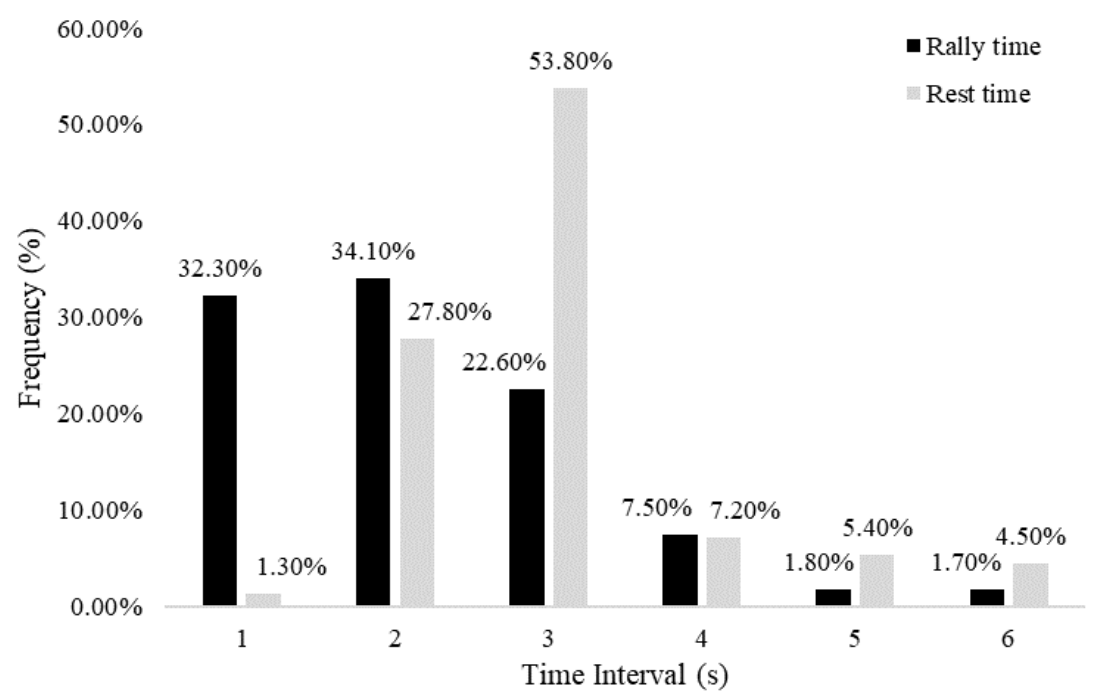

Abbreviations: Data as a mean percentage of three badminton simulation matches.

Figure 3. The mean percentage of playing intervals (performance time) and recovery (rest time) of three matches 


\subsection{Heart Rate and Lactate}

Table 5 and Figure 4 display the lactate and heart rate measures in the laboratory and on-court conditions, respectively. For the lactate measures, a significant increase was observed from pre-test to post-test in both conditions $\quad(p=0.001)$. Furthermore, ANOVA also determined the significant main effects of time $(p=0.001)$ and group $(p=0.001)$. Average heart rate results aligns with the lactate results of participants, where independent t-test revealed significant HR average value among laboratory and on-court conditions ( $p=0.001)$.

Table 5. Different in lactate measures of all participants in the laboratory and on-court conditions

\begin{tabular}{ccccccccc}
\hline \multirow{2}{*}{ Variables } & \multicolumn{3}{c}{ Laboratory condition } & \multicolumn{3}{c}{ On-court condition } & \multicolumn{3}{c}{ Anova $p$-values $^{(\mathrm{a})}$} \\
\cline { 2 - 9 } & Pre & Post & $p^{(\mathrm{b})}$ & Pre & Post & $p^{(\mathrm{b})}$ & Group & Time \\
\hline Lactate $(\mathrm{mmol} / \mathrm{L})$ & $2.69 \pm 1.15$ & $12.30 \pm 3.53$ & $0.001^{*}$ & $3.05 \pm 1.13$ & $4.6 \pm 1.11$ & 0.007 & $0.001^{*}$ & $0.001^{*}$ \\
\hline
\end{tabular}

Abbreviations:

The values are presented as mean $\pm \mathrm{SD}$.

(a)Superscript: A $2 \times 2$ repeated measures analysis of variance (ANOVA). *Values are significantly different between laboratory and on-court conditions $(\mathrm{p}<0.05)$.

(b) Superscript: Independent t-test. *Values are significantly different between laboratory and on-court conditions

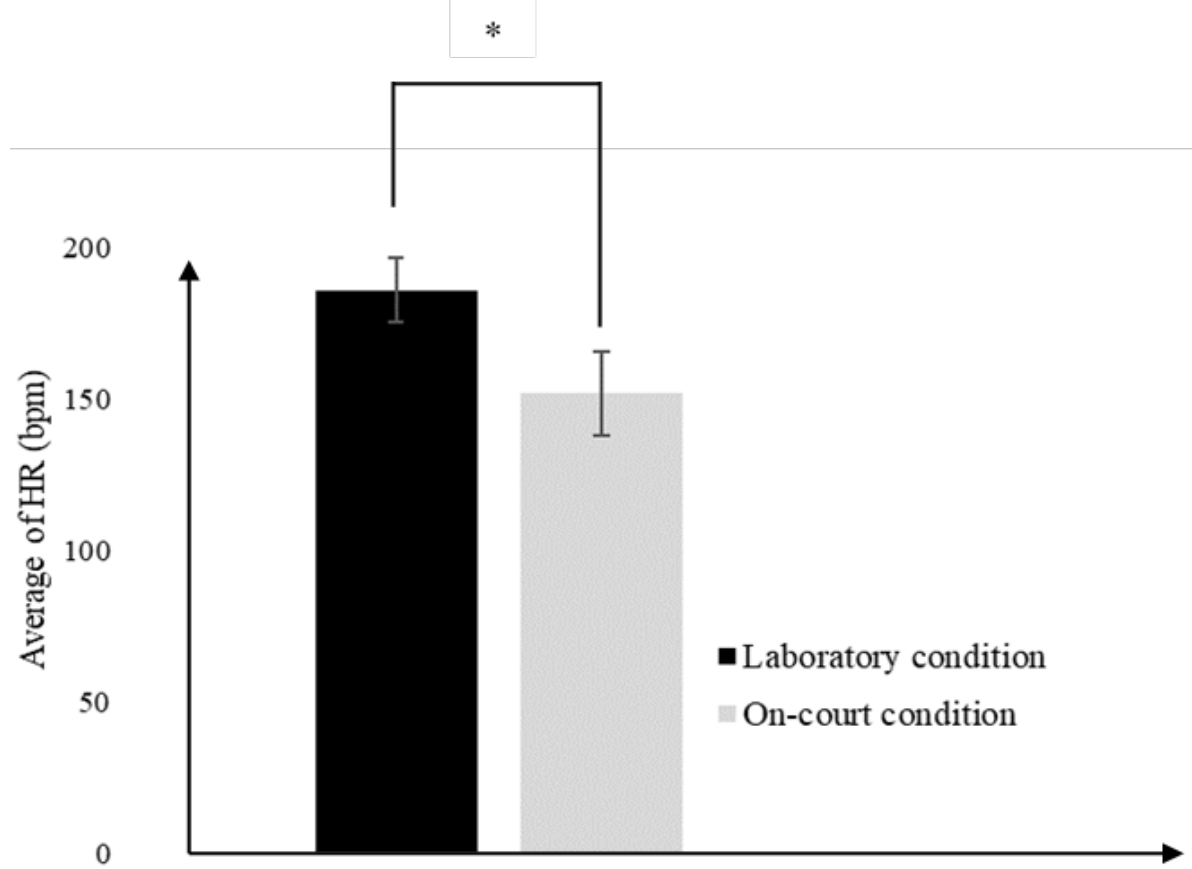

Abbreviations:

The values are presented as mean $\pm \mathrm{SD}$.

* Values are significantly different between laboratory and on-court conditions $(\mathrm{p}<0.05)$.

Figure 4. Specific different in HR average in the laboratory and on-court conditions 


\section{Discussion}

The aim of this study is to assess the physiological characteristics of Indonesian junior badminton players and to use the findings to devise effective training methods with greater precision. To the author's best knowledge, this is the first study that assessed the physiological characteristics of Indonesian junior badminton players, specifically men's doubles category. With respect to the goal of this study, it succeeded in revealing some very useful results for trainers, sport researchers, and athletes. The main findings of this study are: (1) determination of the average of $\mathrm{VO}_{2 \max }$ and anthropometric characteristics of Indonesian men's doubles category; (2) badminton is a sport that is characterized by intermittent activities of high and low intensities, interspersed by short recovery periods, and; (3) the average of heart rate and blood lactate measurements in laboratory condition are significantly greater than the measurements obtained during the on-court condition.

In the present study, the average height of Indonesian junior badminton athletes playing in the men's doubles category is $175.3 \mathrm{~cm}$; the average body weight is $69.01 \mathrm{~kg}$; the average body fat is $13.02 \mathrm{~kg}$; and, the average BMI is found to be $22.43 \mathrm{~kg} \mathrm{~m}^{-2}$. These findings are supported by previous studies [2,23]. For example, Phomsoupha et al. [2] explained that the average height of badminton athletes from the Asian region (Indonesia, Malaysia, Turkey), and Spain is shorter (mean $171 \mathrm{~cm}$ ) than badminton athletes from the regions of Denmark, Germany, the Czech Republic, and South Africa (mean $182 \mathrm{~cm}$ ). On the other hand, a significant development is observed in relation to the anthropometry (height and weight) of Indonesian junior badminton athletes. This is evident from the increase in height and weight that has been evaluated by previous studies. Rahmawati et al. [24] revealed that the height and weight of junior Indonesian badminton athletes in 2007 were an average of $160.4 \mathrm{~cm}$ and $48.7 \mathrm{~kg}$ respectively, whereas this study conducted in 2019 revealed that the average height and weight Indonesia's junior badminton athletes are $175.3 \mathrm{~cm}$ and $69.01 \mathrm{~kg}$ respectively. Previous research conducted by Larson et al. explained that nutritional factors, level of play, and training characteristics are some of the factors that can influence anthropometric changes in athletes.

During the match analyses, it was observed that the double Indonesian junior badminton athletes hit drive (466) and drop (337) shots the most often. Although some difficulties were encountered while finding supportive literature related to the playing characteristics of men's doubles badminton athletes, the findings of this study appear to be similar in measurements to junior badminton athletes studied by Ming et al. In their research, Ming et al. [25] explained that junior badminton athletes often take drop shots and drive shots. When observed and compared, the findings in both studies actually differ. Responding to these differences, existing literature revealed that each region has its own characteristics in determining their playing style [11]. The reason for this occurrence is that each region has a different concept of exercise and physiological characteristics. Furthermore, due to the fact that this study is the first finding that specifically reveals the characteristics of Indonesian badminton athletes in the men's doubles category, further research is needed that can confirm and compare badminton athletes in the men's doubles category belonging to Indonesia and badminton athletes in the men's doubles category belonging to other nationalities.

This study shows several phenomena that establishes a connection between the influence of anthropometry and athlete's performance when competing. This is evident from the fact that the number of shots performed by pair 6 was significantly less when compared to other pairs. On careful analysation, pair 6 is found to have shorter anthropometric characteristics and tends to be lean compared to other pairs. Although the correlation between anthropometry and performance quality was not studied using statistical analysis, there was de facto an influence of anthropometry on athlete performance when competing. This can also be explained by using existing literature, which was stated by Ismail et al. [26] He explained that the role and relationship between anthropometry, skill ability and physical performance, are three important characteristic components that must be achieved in gaining success in sports.

The match analyses reinforces the fact that badminton is a racquet sport that has characteristics of intermittent activities of high and low intensities, interspersed by short recovery periods [3]. This is evident from the results found, wherein the majority (89\%) of rally time occurred between 3-11 seconds during the 3 matches held. At the same time, $81.6 \%$ of rest intervals were mostly situated between 4-11 seconds. The results of this study were supported by several previous studies [15,27]. For example, Faude et al. [15] also asserted the fact that badminton is a racquet sport characteristic of intermittent activities of high and low intensities, interspersed by short recovery periods. This is further proved through research, which says that $86.7 \%$ of rallies occur in less than 9 seconds, and $87.1 \%$ of the rest time occurs in the range of 6-15 seconds. On the other hand, previous literature has already confirmed that, in badminton, the aerobic system provides $65 \%$ of the energy to recover in a short time during each rally match which consists of movements with low intensity characteristics, and continuous alternation of high intensity rallies [28], while anaerobic system supplies energy mainly during the explosive movements of each rally.

Another phenomenon that is observed during this study is the high concentrations of blood lactate and average heart rate of athletes, when tested in the laboratory condition and on-court condition and compared. The results were found to be in line with several previous 
studies that used the same concept, namely the comparison of laboratory and on-court tests [15,17]. For example, Rampichini et al. [17] found a high yield of blood lactate and average heart rate of badminton athletes on performing the incremental exercise test in the laboratory compared to the match simulation test on-court. The study explained that the existence of an adrenergic strategy that results from stress due to the emergence of a test, requires the athletes to develop speed, accuracy, and a high level of concentration when undertaking exercise incremental tests in the laboratory. Because of the similarity in the protocol characteristics between this study and the previous study, the findings in this study can be considered as additional findings which reinforce that the adrenergic strategy is indeed a sympathetic activity response, which is involved in the process of the incremental exercise test, that causes lactate and HR levels of the athletes to be higher when compared to the on-court match simulation test.

This study has established several facts related to physiological and match characteristics of Indonesian junior badminton athletes playing the men's doubles category. Coaches and sports researchers are hence encouraged to utilize the findings of this study such as the concept of high intensity interval combined agility training in order to develop the athlete's potential for success in every tournament and competition. For example, Laurerio et al. employed the Badcamp test as the method for improving endurance and agility in badminton players. In the findings, Laurerio et al. explained that the Badcamp test requires every badminton athlete to release their agility skills accompanied by high intensity for the successful completion of the test. Apart from the Badcamp test, there exists several other measurement methods that comprise of high intensity intervals, which can be used by coaches or badminton athletes to improve their performance [29-31].

The research is bound to have some limitations. Firstly, the number of participants in this study is strictly small, and future studies on the same theme are encouraged to use bigger samples, especially for badminton athletes in Indonesia in order to discover the phenomena that have not been covered by this study. Secondly, more optimal results can be achieved in future studies during the on-court condition test. Efficient equipment can be utilized, including the portable metabolic devices that can assess physiological profile on court. Thirdly, this study does not focus on the measurement of the statistical correlation between the effect of anthropometry and competition performance. This leaves room for further research, to measure this correlation, and strengthen and vividly explain in more detail the findings of this study. More or less, some of the results and facts used in this study that have been realized are expected to help coaches, athletes or sport researchers to find and develop training concepts that are favourable to the development of athletes or act as the right formula for screening the talents of potential athletes.

\section{Conclusion}

The results of this study provided evidence and demonstrated that physiological characteristics of badminton men's doubles category are intermittent activities of high and low intensities, interspersed by short recovery periods. It was also established that drive shots were used more frequently by Indonesian badminton men's doubles athletes. On the other hand, adrenergic strategy plays a major role in enhancing the levels of HR and lactate in laboratory settings than that on-court conditions. Utilizing all the findings, practical advice can be devised to coaches of junior badminton athletes. Specifically, the use of high-intensity interval combined agility training is encouraged as a design training in order to improve the athlete's performance.

\section{Acknowledgment}

The authors would like to thank Rumah Sakit Olahraga Nasional (RSON) and Jaya Raya Badminton Club for providing data for the study.

\section{Declaration of Conflicting Interests}

The authors state no conflict of interest with respect to the research, authorship, and/or publication of this article.

\section{Funding}

The authors would like to thank the Badminton World Federation (BWF) who funded the project (BWF Research Grants 2019).

\section{REFERENCES}

[1] Abia'n P., Castanedo A., Feng X.Q., Sampedro J., Abian-Vicen J, "Notational comparison of men's singles badminton matches between Olympic Games in Beijing and London,” Int J Perform Anal Sport, vol. 14, pp. 42-53, 2014. DOI: $10.1080 / 24748668.2014 .11868701$.

[2] Phomsoupha M., Laffaye G, “The Science of Badminton: Game characteristics, anthropometry, physiology, visual fitness and biomechanics" Sports Med, vol. 45, no. 4, pp. 473-495, 2015. DOI: 10.1007/s40279-014-0287-2.

[3] Jeyaraman R., District E., Nadu T, "Prediction of playing ability in badminton from selected anthropometrical physical and physiological characteristics among inter 
collegiate players,” Int J Adv Innov Res, vol. 2, no. 3, pp. 11, 2012.

[4] Junaidi J., Apriantono T., Winata B., Hasa F., Apriyanto T., Syahruddin S, "Effects of cinnamon extract supplementatio n on creatine kinase activity in badminton athletes," Human Movement, vol. 21, no. 4, 2020. DOI: https://doi.org/10.5114/hm.2020.94196.

[5] Alcock A., Cable N.T. "A comparison of singles and doubles badminton: heart rate response, player profiles and game characteristics. Int J Perform Anal Sport,” vol. 9, no. 2, pp. 228-37, 2009. DOI: https://doi.org/10.1080/2474866 8.2009.11868479.

[6] Liddle S.D., Murphy M.H., Bleakley W, “A comparison of the physiological demands of singles and doubles badminton a heart rate and time/motion analysis," J Hum Mov Stud, vol. 29, pp. 159-79, 1996.

[7] Heller J, "Physiological profiles of elite badminton players aspects of age and gender," Br J Sports Med, vol. 44, no. 17, pp. 1S-13S, 2010. DOI: 10.1136/bjsm.2010.078725.51.

[8] Singh J., Raza S., Mohammad A, "Physical characteristics and level of performance in badminton: a relationship study,” J Educ Pract, vol. 2, no. 5, pp. 6-10, 2011.

[9] Lee J.J.J., Loh W.P, “A State-of-the-art review on badminton lunge attributes,” Comput Biol Med, vol. 108, pp, 213-222, 2019, DOI: 10.1016/j.compbiomed.2019.04.0 03.

[10] Ooi C.H., Tan A., Ahmad A., Kwong K.W., Sompong R., Ghazali K.A.M., et al, "Physiological characteristics of elite and sub-elite badminton players", J Sports Sci, vol.27, no. 14, pp. 1591-1599, 2009, DOI:10.1080/0264041090335290 7.

[11] Cabello D., Padial P., Lees A., Rivas F, “Temporal and physiological characteristics of elite women's and men's singles badminton,” Int J Appl Sport Sci, vol. 16, no. 2, pp. 1-26, 2004.

[12] Chin M.K., Wong A.S., So R.C., Siu O.T., Steininger K., Lo D.T, "Sport specific fitness testing of elite badminton players,” Br J Sports Med, vol. 29, no. 3, pp.153-157, 1995, DOI: 10.1136/bjsm.29.3.153.

[13] Jaworski J., Żak M., Lech G., Bujas P., Żak S., Tchórzewski D, "Somatic and functional compensations in junior badminton players,” Human Movement, vol. 19, no. 1, pp. 26-33, 2018, DOI:10.5114/hm.2018.73609.

[14] Nadzalan A., Mohamad N., Lee J., Chinnasee C, "Relationship between muscle architecture and badminton-specific physical abilities,” Human Movement, vol. 19, no. 1, pp. 44-50, 2018, DOI:10.5114/hm.2018.7361 1.

[15] Faude O., Meyer T., Rosenberger F., Fries M., Huber G., Kindermann W, "Physiological characteristics of badminton match play,” Eur J Appl Physiol, vol. 100, no. 4, pp. 479-485, 2007. DOI: 10.1007/s00421-007-0441-8.

[16] Cabello Manrique D., González-Badillo J.J, “Analysis of the characteristics of competitive badminton,” Br J Sports Med, vol. 37, no. 1, pp. 62-66, 2003. DOI: 10.1136/bjsm.37.1.62.

[17] Rampichini S,. Limonta E., Pugliese L., Cè E., Bisconti
AV., Gianfelici A., et al, "Heart rate and pulmonary oxygen uptake response in professional badminton players: comparison between on-court game simulation and laboratory exercise testing,” Eur J Appl Physiol, vol. 118, no. 11, pp. 2339-2347, 2018. DOI: 10.1007/s00421-018-39 60-6.

[18] Jaworski J., Żak M, "Identification of determinants of sports skill level in badminton players using the multiple regression model," Human Movement, vol. 17, no. 1, pp. 21-28, 2016. DOI:10.1515/humo-2016-0004.

[19] Farah Layth Naji., Tengku Fadilah Tengku Kamalden., Saidon Amri., "Psychological Distress and Physiological Markers: Differences Comparison in First Division Football Players," International Journal of Human Movement and Sports Sciences, Vol. 8, No. 1, pp. 43 - 49, 2020. DOI: 10.13189/saj.2020.080106.

[20] Foster C, "Monitoring training in athletes with reference to overtraining syndrome,” Med Sci Sports Exerc, vol. 30, no. 7, pp. 1164-1168, 1998, DOI: 10.1097/00005768-1998070 00-00023.

[21] Nieman DC., Austin MD., Dew D., Utter AC, "Validity of COSMED's quark CPET mixing chamber system in evaluating energy metabolism during aerobic exercise in healthy male adults,” Res Sports Med. Vol. 21, no. 2, pp. 136-145, 2013. DOI:10.1080/15438627.2012.757227.

[22] Gore CJ., Catcheside PG., French SN., Bennett JM., Laforgia J, “Automated $\mathrm{VO}_{2 \max }$ calibrator for open-circuit indirect calorimetry systems,” Med Sci Sports Exerc, vol. 29, no. 8, pp. 1095-1103, 1997. DOI:10.1097/00005768-19 9708000-00016.

[23] Hussain S, "Somatotype and body composition of adolescent badminton players in Kerala,” Int J Adv Sci Tech Res, vol. 6, no. 3, pp. 105-111, 2013.

[24] Rahmawati N.T., Budiharjo S., Ashizawa K, "Somatotypes of young male athletes and non-athlete students in Yogyakarta, Indonesia,” Anthropol Sci, vol. 115, no. 1, pp. 1-7, 2007.

[25] Ming C.L., Keong C.C., Ghosh A.K, "Time motion and notational analysis of 21 point and 15-point badminton match play,” Int J Sport Sci Eng, vol. 2, no, 4, pp. 216-222, 2008.

[26] Ismail M.S.H., Boon Suen A., Othman C.N., Singh., R, "Comparison of anthropometric variables and leg strength of volleyball, basketball and badminton players,” Malays J Med Lab Sci, vol. 10, no. 1, pp. 26-30, 1993.

[27] Abian-vicen J., Castanedo A., Abian P., Sampedro J, "Temporal and notational comparison of badminton matches between men's singles and women's singles,” Int J Perform Anal Sport, vol. 13, no. 2, pp. 310-320, 2013. DOI: 10.1080/24748668.2013.11868650.

[28] Wonisch M., Hofmann P., Schwaberger G., von Duvillard S.P., Klein W, "Validation of a field test for the non-invasive determination of badminton specific aerobic performance,” Br J Sports Med, vol. 37, no. 2, pp. 115-118, 2003. DOI: 10.1136/bjsm.37.2.115.

[29] de França Bahia Loureiro L.Jr, Costa Dias M.O., Cremasco F.C., da Silva M.G., de Freitas P.B, "Assessment of Specificity of the Badcamp Agility test for Badminton 
Players,” J Hum Kinet, vol. 57, pp. 191-198, 2017. DOI: 10.1515/hukin-2017-0060.

[30] Madsen CM., Højlyng M., Nybo L, “Testing of Badminton-Specific Endurance," J Strength Cond Res, vol. 30, no. 9, pp. 2582-2590, 2016. DOI:10.1519/JSC.0000000
000001350.

[31] Madsen CM., Karlsen A., Nybo L, "Novel speed test for evaluation of badminton-specific movements," J Strength Cond Res, vol. 29, no. 5, pp. 1203-1210, 2015. DOI:10.1519/JSC.0000000000000635. 